Mini Review

\title{
Dualistic face of $H$. pylori and some herpes viruses co-infection observation from clinical practice
}

\begin{abstract}
Herpes viruses as well as $H$. pylori share some common characteristics. They are causing almost ubiquitous infections, establish lifelong latency and are considered in the carcinogen-sis of different malignancies. The philosophy of the host-pathogen interaction is multifaceted, and not very well studied. Despite the fabulous progress in molecular research, there are some simple observations made in routine clinic that still are not explained but raised attention of the physicians. We present an observation among adults who attended outpatient clinic. We found that the prevalence ratio of $H$. pylori is significantly smaller in case of high titers of EBV antibodies. Similarly, subjects with acute HSV1 or HSV2 infection were less prone to be positive for $H$. pylori. The question of $H$. pylori might have a protective role for herpes infections or immune responses to heresies prevent development of $H$. pylori infection remains unclear.
\end{abstract}

Keywords: herpes viruses, EBV, HSV, helicobacter pylori
Volume 6 Issue 4 - 2017

\author{
Assya Krasteva,' Mihaela Petrova ${ }^{2}$ \\ 'Department of Oral and Imaging Diagnostics, Medical \\ University, Bulgaria \\ ${ }^{2}$ BalkanMed Medical Center, Bulgaria
}

Correspondence: Mihaela Petrova, BalkanMed Medical Center, Bulgaria,Email mpetrova@gmail.com

Received: February 23, 2017 | Published: March 30, 2017

\section{Introduction}

Epstein - barr virus (EBV) causes a ubiquitous infection worldwide affecting more than $90 \%$ of the adult population. ${ }^{1}$ It is due to its ability to establish lifelong latency with episodes of reactivations with lack of major clinical symptoms in majority of cases. EBV is a member of Herpesviridae, sharing the main characteristics of the herpes infections - persistency, latency and reactivation. It has been linked to a variety of malignancies and some data show that EBV reactivations might change the natural history of other concomitant infections. ${ }^{2}$ Persistent herpes infections including EBV might augment the immune senescence, having direct imply cation on longevity. ${ }^{3}$ What are more interesting is the clinical implications of concomitant and consecutive infections and reactivations of EBV and other pathogens. It is believed that concomitant infection is generally possible due to similar pathways and is associated with more severe course of the disease.

The consecutive infective agent however could find the host in immune competent state. EBV generally attacks the whole immune system and usually leads to stable immune response. It has been speculated that EBV infection might have a protective role ${ }^{4}$ for other types of infections. The same dispute can be seen in the literature about the role of Helicobacter pylori. There are quite a few studies showing the protective role of non-eradicated $H$. Pylori infection in different autoimmune diseases. ${ }^{5}$ Could this be the scenario with HSV? We are presenting two hypotheses about potential protective role of the current H. Pylori infection against manifestation of herpes infection.

We have tested sera of total 190 consecutive dental patients (mean age 52, SD 12, 45 males) who presented in the outpatient clinic. All the patients underwent oral examination. The clinical findings included common oral pathology and superficial oral lesions. Maxillofacial malignancy was excluding criteria as well as diagnosis of other malignancy. All of the patients signed informed consent to participate in this observational study.

\section{EBV and H. pylori}

We have measured serological responses to define the EBV and $H$. pylori infection. Not surprisingly, over $90 \%$ of the participants had evidences of past infection. Very high titer $(>750 \mathrm{U} / \mathrm{ml})$ of EBV VCA $\operatorname{IgG}$ had $27 \%$ of them. In 64 of the participants we did the simultaneous serology test for H.pylori, which showed up positive in $55 \%$ of cases $(35 / 64)$.

Interestingly, in the group with EBV VCA IgG lower than $750 \mathrm{U} / \mathrm{ml}$, 31 were positive for $H$. Pylori and 17 were negative. On the contrary, in the group of higher EBV VCA (over 750U/ml) less participants were positive (four) than negative (twelve) for H.pylori ( $\mathrm{p}=0.0087$, Fisher's), Figure 1.

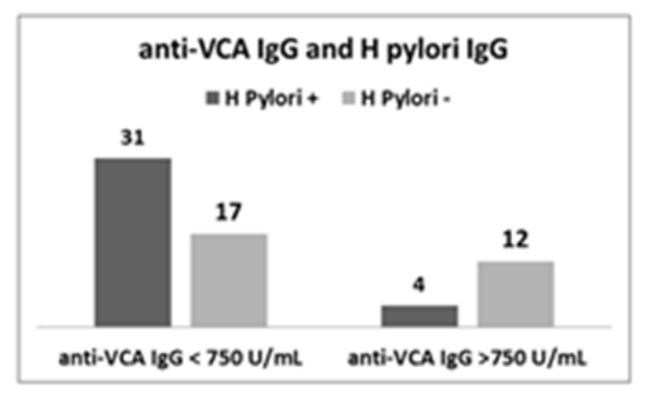

Figure I Anti-VCA IgG and H. pylori.

We may speculate that in people with strong humoral immune response to EBV the H.pylori infection is less common - $25 \%$ vs $65 \%$ $(\mathrm{p}<0.01)$.

\section{HSVI/2 and H. pylori}

Among 126 participants we have detected serology signs for acute HSV infection in $18 \%$. Positive for H.pylori were $56 \%$ of 88 tested regardless age (mean 52, SD 12) and sex.

We have tested for H.pylori half of the participants. The results showed that general serology prevalence of the HSV was $13 \%$ and of the H.pylori - 57\%. Participants without evidences for HSV2 were tested positive for H.pylori in $63 \%$ of cases, while none of the positive for HSV2 was also positive for H.pylori ( $\mathrm{p}=0.0046$, Fisher's). We noticed that acute HSV2 infection is detected more often in negative for H.pylori persons, Figure 2. 


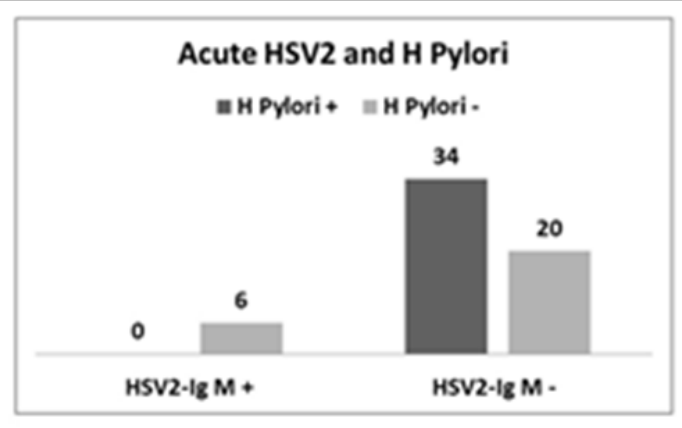

Figure 2 Acute HSV2 and H. Pylori.

The same pattern is apparent when comparing HSV1 acute vs lack of HSV infection. Subjects with acute infection were less prone to be positive for H.pylori (1/8 vs 33/52, p=0.0164, Fish-er's) (Figure 3 ), There is an open question if H.pylori might play protective role against other infections. That question has been raised in some previous papers focused on local interactions between viruses and bacteria in gastric mucosa. It has been shown that peptic ulcer patients who were positive for HSV-1 were more likely to be negative for $H$. pylori. Furthermore, authors found an inverse association between HSV-1 and H. pylori infection in Greek adult population. ${ }^{6}$ On the contrary, another study among US adolescents revealed that HSV-1 seropositivity is associated with a higher H. pylori seroprevalence. The study was not able to found biological reasons and concluded that shared routes of transmission might explain the observation (7). Our data showed the opposite association, which could be consequence of local immune interactions or kind of more sophisticated interplay of the host immune responses.

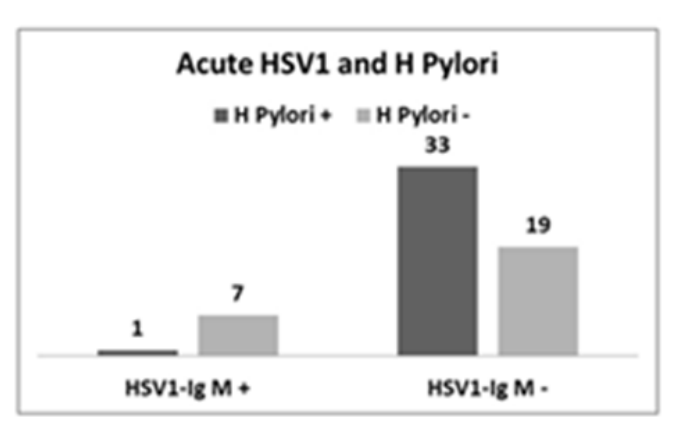

Figure 3 Acute HSVI and H. Pylori.

H. pylori and EBV co-infection attracted a lot of attention in the recent decade. Quite a few papers commented the role of EBV in the gastric carcinogenesis, in which H.pylori is a major player. Less understood is the participation of EBV in chronic gastric inflammation, but some studies argue that EBV, similar to and together with Helicobacter pylori, is an early participant in the GC oncogenic process through promoting chronic inflammation and increased tissue damage. ${ }^{8}$ Both pathogens share similar patterns of cell signaling pathways leading to proliferation, increased cell survival, immune evasion. Recent paper by Saju et al., ${ }^{9}$ showed that host's SHP1 phosphatase antagonizes Helicobacter pylori CagA and can be down regulated by Epstein-Barr virus. In vitro infection of gastric epithelial cells with EBV induces SHP1 promoter hypermethylation, which strengthens phosphorylation-dependent
CagA action via epigenetic down regulation of SHP1 expression. ${ }^{9}$ While H. pylori cause epigenetic silencing of tumour-suppressor genes to deregulate cellular pathways, EBV-positive tumors exhibit a widespread and distinctive DNA hypermethylation profile. ${ }^{10}$ Beyond the gastric cancer, the interaction between EBV and H.pylori is in the focus of recent research on peptic ulcer disease. Serology study from Mexico found that peptic ulcer was associated with high antiEBV IgG titers, while anti-EBV IgA was positively associated with gastric ulcer. The EBV reactivation in gastric and duodenal epithelium increased the risk to develop ulcer up to ten-fold. ${ }^{11}$ our results actually supposed opposite association between H.pylori and EBV - high titers of EBV increases the likelihood of H.pylori negativity. This might be explained by augmented local immune responses as shown in the study of Cárdenas-Mondragón et al., ${ }^{12}$ Our findings suppose that clinicians should have broader approach to H.pylori infection as it seems to be longtime human companion. The current test and treat strategy for H.pylori might need more research focused on coexistence of other infections. Our results has to be commented considering some limitations as relatively small numbers of the participants, lack of consensus for serology criteria for EBV reactivation and the possibility of serology interference. We believe however, that interactions between pathogens have to be further studied as its consequences are important not only for the basic science lab but also for the routine clinical practice. The characteristics of H.pylori and herpes viruses co-infections need to be explored more profound beyond the carcinogenesis but in more frequent gastrointestinal benign diseases. The question of H.pylori might have a protective role for herpes infections or immune responses to herpeses prevent development of H.pylori infection remains unclear.

\section{Acknowledgments}

None.

\section{Conflicts of interest}

Author declares there are no conflicts of interest.

\section{Funding}

None.

\section{References}

1. Balfour HH, Sifakis F, Sliman JA, et al. Age-specific prevalence of Epstein-Barr virus infection among individuals aged 6-19 years in the United States and factors affecting its acquisition. $J$ Infect Dis. 2013;208(8):1286-1293.

2. Hsu JL, Glaser SL. Epstein-barr virus-associated malignancies: epidemiologic patterns and etiologic implications. Crit Rev Oncol Hematol. 2000;34(1):27-53.

3. Wang $\mathrm{C}$, Liu Y, Xu LT, et al. Effects of ag-ing, cytomegalovirus infection, and EBV infection on human B cell repertoires. J Immunol. 2014;192(2):603-611.

4. Petrova M, Kamburov V. Epstein-Barr virus: Silent companion or causative agent of chronic liver disease? World $J$ Gastroenterol. 2010;16(33):4130-4134

5. Hasni SA. Role of Helicobacter pylori infection in autoimmune diseases. Curr Opin Rheumatol. 2012;24(4):429-434.

6. Tsamakidis K, Panotopoulou E, Dimitroulopoulos D, et al. Herpes simplex virus type 1 in peptic ulcer disease: an inverse association with Helicobacter pylori. World J Gastroenterol. 2005;11(42):6644-6649. 
7. Baccaglini L, Schoenbach VJ, Poole C, et al. Wiesen CAsso-ciation between herpes simplex virus type 1 and Helicobacter pylori in US adolescents. Oral Surg Oral Med Oral Pathol Oral Radiol Endod. 2016;101(1):63-69.

8. Fuentes-Pananá EM, Morales-Sánchez A. Epstein-Barr Virus-associated Gastric Cancer and Potential Mechanisms of Oncogenesis. Curr Cancer Drug Targets. 2016.

9. Saju P, Murata-Kamiya N, Hayashi T, et al. Host SHP1 phosphatase antagonizes Helicobacter pylori CagA and can be downregulated by Epstein-Barr virus. Nat Microbiol. 2016;14(1):16026.
10. Wu WK, Yu J, Chan MT, et al.Combinatorial epigenetic deregulation by He-licobacter pylori and Epstein-Barr virus infections in gastric tumourigenesis. J Pathol. 2016;239(3):245-249.

11. Cárdenas-Mondragón MG, Torres J, Flores-Luna L, et al. Epstein-Barr virus association with peptic ulcer disease. Anal Cell Pathol (Amst). 2015;2015:164840.

12. Cárdenas-Mondragón MG, Torres J, Flores-Luna L, et al. Case-control study of Epstein-Barr virus and Helicobacter pylori serology in Latin American patients with gastric disease. Br J Cancer. 2015;112(12):18661873 